\title{
Localization of Optic Disc and Blood Vessel Segmentation: A Survey
}

\author{
Bhavya K. Bharathan \\ M.Tech Student \\ Department of CSE \\ APJ Abdul Kalam \\ Technological University
}

\author{
Anjali K. \\ M.Tech Student \\ Department of CSE \\ APJ Abdul Kalam \\ Technological University
}

\author{
Remya P. S. \\ Assistant Professor \\ Department of CSE \\ Vidya Academy of Science \\ \&Technology
}

\begin{abstract}
Nowadays, number of retinal disorders are affecting to all age groups. Various research activities are being carried out to diagnosis the disorders. Damage to retina leads to loss of vision. An efficient detection of optic $\operatorname{disc}(\mathrm{OD})$ is an important task in an automated retinal image examination system. Most of the OD detection algorithms are developed for normal retinal images. So, to detect OD from normal, healthy and abnormal retinal images are challenging tasks. In this work, the various methods used to locate OD and blood vessel segmentation from retinal images are discussed.
\end{abstract}

\section{General Terms}

Retina, Morphology, Fundus images, Blood vessels

\section{Keywords}

Vessel Segmentation, OD Localization, Thresholding

\section{INTRODUCTION}

Blood vessels in the retinal images is part of the retina that serves to provide blood and oxygen to the blood vessel of the retina. If the blood and oxygen supplies are not smooth, then this may be a indication to discover whether there are health problems (hypertension, cardiovascular, stroke or diabetes). To discover these problematic veins that can be done with the segmentation of blood vessels in retinal digital images. To screening diseases like glaucoma, optic disk segmentation is the major task.

Segmentation of retinal image structures has been of huge interest because it could be used as a nonintrusive identification in modern ophthalmology. The morphology of the retinal blood vessel and the optic disk is an important structural sign for assessing the occurrence and harshness of retinal diseases such as diabetic retinopathy, hypertension, glaucoma, hemorrhages, vein occlusion, and neovascularization. However, to evaluate the diameter and crookedness of the retinal blood vessel or the shape of the optic disk, manual planimetry has commonly been used by ophthalmologists, which is time consuming and there is a chance to occur error when a large number of images are acquired to be labeled by hand. Therefore, a reliable automated method for retinal blood vessel and optic disk segmentation, which conserve various characteristics of vessels and optic disk is take more attention in computer aided diagnosis(CADx).

\subsection{Eye Anatomy}

Basically the human eye is an organ which reacts to light for many different purposes and is made up of three coats, enclosing three transparent structures. The outermost layer is consists of the cornea and sclera. The middle layer composed of the choroid, ciliary body and iris. The innermost is the retina. The light rays from an object are reflected off and enter through the cornea. It then refracts the rays that pass through the pupil. Surrounding the pupils is the iris, the colored portion of the eye. The pupil opens and closed to regulate the amount of light passing through it. Hence, we are able to see the dilation of the pupils. Light rays will overtake the lens that is located behind the pupils. This lens alter the shape of the rays by further bending and focusing them to the retina located at the back of eyes.

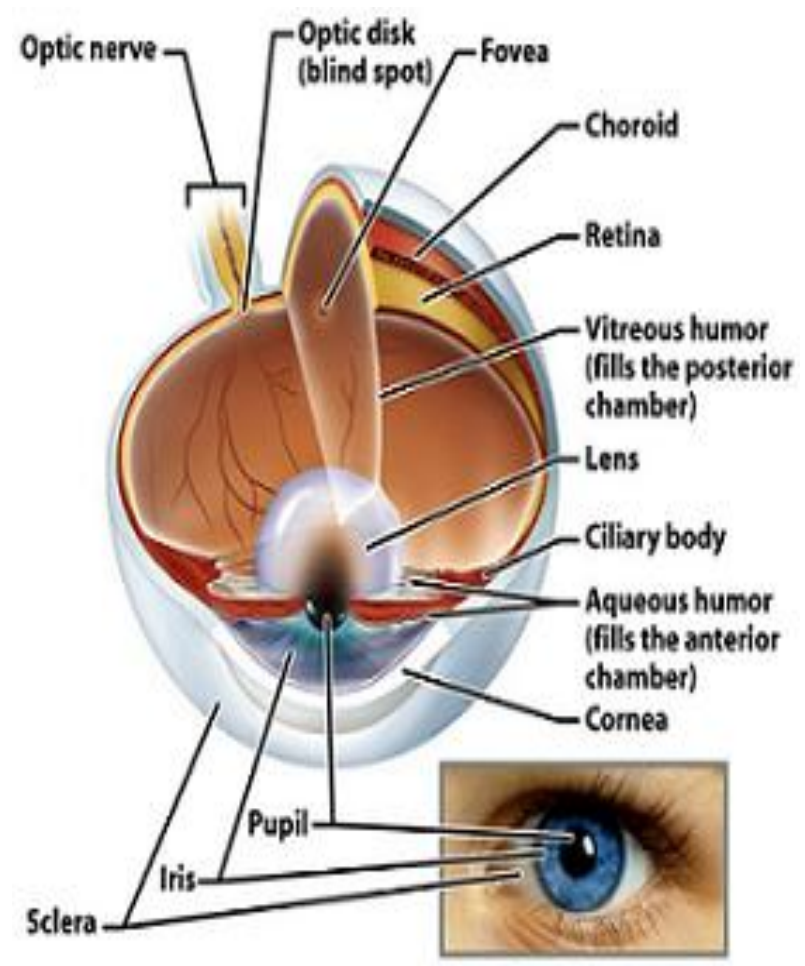

Figure 1: Simple diagram of the parts of the eye

The retina consists of two major types of light-sensitive receptors which are also called tiny light-sensing nerve cells. They are cones nerve cells and rods nerve cells. The cones enable us to see bright light which produces photonic vision. Cones are mostly concentrated in and near the fovea. Only a few are present at the sides of the retina. It provides a clear and sharp image vision as when one looks at an object directly. It discovers colors and detects fine details. The rods however, enable us to see in the dark which produces isotopic vision. It detects motion in the dark. It is located outside the macula and goes all the way to the outer of edge retina. Rod density is greater in the peripheral retina than in the central retina. Hence it provides side vision. Cones and rods are connected through intermediate cells in the retina to nerve 
fibers of the optic nerve. When rods and cones are stimulated by light, the nerves sendoff impulses to the brain through these fibers. Figure 1[25] illustrate a simple diagram of the parts of the eye.

\section{MATERIALS}

Assessment of the segmentation techniques can be done using the publically accessible 6 datasets DRIVE [1], STARE [2], DIARETDB0[3], DIARETDB1[4], CHASE DB1[5] and MESSIDOR[6]. Both the databases contains test and training images, where training images are the ground truth images which are by hand segmented by the experts. These databases are widely used by various researchers for the evaluation of their results. Some of the techniques used both the databases for estimation purposes. Very few techniques are applied on random images. Datasets that can be used for OD localization is as shown in Table 1.

Table 1. Database used for OD localization

\begin{tabular}{|c|c|c|}
\hline Sr. No. & Test Datasets & No. of images \\
\hline 1 & DRIVE & 40 \\
\hline 2 & STARE & 81 \\
\hline 3 & DIARETDB0 & 130 \\
\hline 4 & DIARETDB1 & 89 \\
\hline 5 & CHASE DB1 & 28 \\
\hline 6 & MESSIDOR & 1200 \\
\hline
\end{tabular}

\section{REVIEW OF METHODS}

Study of colour fundus images is considered to be the finest analytic modality available till date as it is dependable, noninvasive and effortless to use. Figure 2 [25] shows the retinal fundus image with major anatomical structures. The retina is an internal surface of eye which acts as the film of eye. An important precondition for automation is the accurate localization of the main anatomical features in the image. An precise and efficient detection of these structures is a significant mission in an automated retinal image analysis system. The OD localization and blood vessel segmentation is important for a lot of reasons. Some of them are mentioned here.

The automatic and well-organized detection of the position of the OD in colour retinal images is an significant and basic step in the automated retinal image analysis system [7], [8].To successfully discover abnormal structures in a retinal image, it is often essential to mask out the usual anatomy from the analysis. An example of this is the OD, an anatomical structure with a bright outward show, which should be ignored when detecting bright lesions. The attributes of OD are alike to attributes of hard exudates in terms of colour and brightness. Hence it is located and removed during hard exudates detection procedure, in that way avoiding false positives. OD detection is the main step at the same time as developing automated screening systems for diabetic retinopathy and glaucoma.

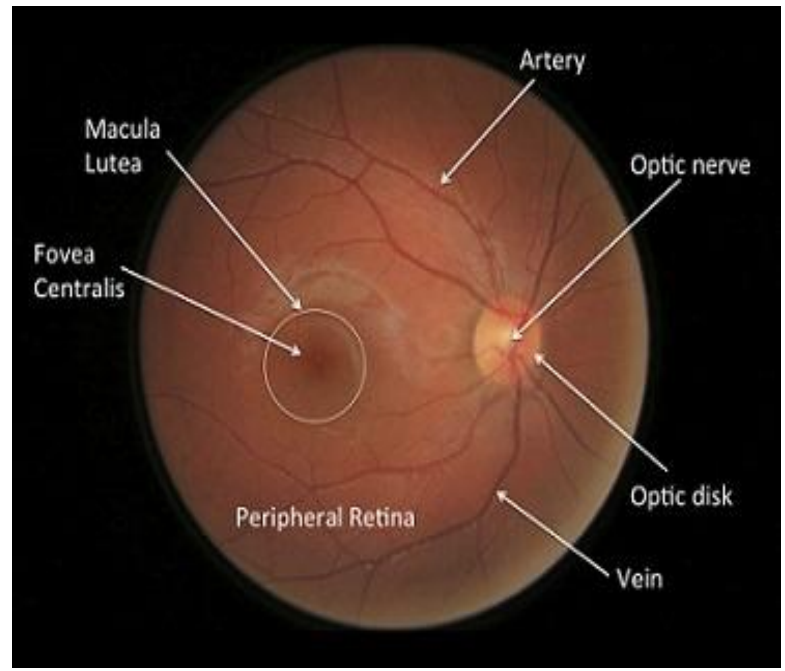

Figure 2: Retinal Fundus Image With Main Anatomical Structures

OD boundary and localization of macula are the two features of retina essential for the detection of exudates and also knowing the harshness of the diabetic maculopathy [9]. In case of diabetic maculopathy lesions recognition, masking the false positive OD region leads to development in the performance of lesion detection.

According to S. Sekhar et al. [10] the optic disk is usually the brightest element on the fundus image, and so a cluster of high intensity pixels will identify the OD location. This method consist of two steps. In the first step, a circular region of interest is found by first separated the brightest region in the image by means of morphological processing, and in the second step, Hough transform is used to discover the main circular feature (corresponding to the optical disk) contained by the positive horizontal gradient image within this region of interest.

Deepali A. Godse et al. [11] presented an automated method for localization of OD. In this, an optimal threshold is obtained through the histogram derived from the source image is scanned from highest intensity value to the lowest intensity value. Apply Threshold and identify bright regions from the retinal images. Select candidate regions which satisfy area criterion and then, Select candidate region which satisfies density criterion. If no candidate region is selected, reduce threshold. If threshold is greater than zero continue the previous steps. After that the centroid of this cluster is determined using calculus method.

Sinthanayothin et al. [12] computed a method to detect the location of the OD by finding the area in the image which has the highest variation in brightness. As the OD frequently appears as a bright disc covered in darker vessels, the variance in pixel brightness is the uppermost there. They also presented method for the detection of center of the macular. They used a template equivalent approach in which the template was a Gaussian blob. The search area was constricted by the information that the macular centre was assumed to be in the darkest part of the image approximately 2.5 times the OD diameter from the OD [13].

Nagveena et al. [14] introduces a user friendly MATLAB based graphical user interface (GUI) that segments the blood vessels using adaptive median thresholding. From the segmented image, the features of blood vessels such as area, 
mean, standard deviation, energy and histogram are calculated, for classification of image as normal or abnormal.

Roychowdhury et al . [15] presented three-stage retinal blood vessel segmentation algorithm. Firstly, a threshold binary image is obtained through high pass filtering and the other binary threshold image is obtained by the reconstruction of the red regions in a green plane image. Then the common regions from the two binary images is extracted and pooled. Secondly, classification is applied to the image obtained after integrating. Lastly, all the pixels classified as vessels are combined with major vessels to get the vascular structure. Further, enhancing of image is performed using postprocessing techniques.

Ishmeet Kaur1 et al. [16] in this, process of segmentation of blood vessels involves three steps i.e. image preprocessing, unsupervised approach and image post-processing. The output of image preprocessing is further given as input to unsupervised learning phase where fuzzy c-means clustering technique is applied to the image which is additionally differentiated by the neutrosophic approach which refine the segmented output by separating the pixels into vessel, nonvessel and indeterminate sets. The post-processing phase includes mathematical morphological operations and final segmented image is obtained.

Franklin et al. [17] proposed a method to recognize the retinal blood vessels with the help of multilayer perceptron neural network. In this procedure, the input is derived from the three colour components, i.e., red, green and blue. By using this method the sight intimidating diseases such as exudates and haemorrhages can be detected. This technique is supervised one so it requires training sets which consist of manually segmented images and image features and further the pixel is classified as a vessel and non-vessel.

Roberto Annunziata et al. [18],An enhanced Hessian-based approach for unsupervised retinal blood vessel segmentation using ad hoc exudate inpainting technique has been described. The application of proposed exudate inpainting technique, followed by a simple enhancement and thresholding method, yields results comparable to state-of-the-art techniques that use specialized, sophisticated enhancement and classification algorithms.

Li et al. [19] presented a model based approach in which an active shape model was used to mine the main course of the vasculature based on the position of the OD. Next, the information from the active shape model was used to discover the macular centre.

Huajun Ying et al. [20] utilized fractal examination to differentiate OD area from other large and bright regions in retinal images due to the reality that the OD area is the converging point of all major vessels. With its location known, segmentation of optic disc can be complete with simple local histogram analysis.

Lalonde et al. [21] proposed a method based on pyramidal decomposition and Handoff-distance based template matching. The green plane of the original image be subsampled and the brightest pixels in this sub-sampled image were chosen as candidate regions. An edge detector was used on the candidate regions in the original image. Next, multiple circular templates be fit to each of the regions by the Hausdorff-distance as a distance measure. The centre of the fitted circular template was taken as the OD centre.
Roychowdhury et al [22] this paper presents a classificationbased automated optic disk segmentation algorithm that detects the OD boundary and location of vessel origin (VO) pixel. First, the green plane of each fundus image is morphologically reconstructed via a circular structuring element whose diameter is half of the average estimated OD diameter corresponding to a particular field of view (FOV). Bright regions are then extracted from the morphologically reconstructed image that lie in close surrounding area of the major blood vessels. Then, bright regions are classified as bright probable OD regions and non-OD regions using region-based features and a Gaussian Mixture Model (GMM) classifier. Also, using joint information based feature ranking strategy, two most discriminating features for bright OD region detection are recognized as the Vessel-Sum within a fixed neighborhood and bright region Solidity. Thus, the bright probable OD region with maximum Vessel-Sum and Solidity is computed as the best candidate region for the OD. Other bright probable OD regions inside 1-disc diameter from the centroid of the best candidate OD region are then discovered as remaining candidate regions for the OD. A convex hull containing all the candidate OD regions is then estimated, and a best-fit ellipse across the convex hull became the segmented OD boundary. Finally, the centroid of major blood vessels within the segmented OD boundary is detected as the VO pixel location.

Adithya et al. [23] proposed the Combaining of two methods, to segment optic disc area both K-means clustering and adaptive morphology methods are used. To detect the optic disc area, k-means clustering method is used and then to segment the optic disk and blood vessel morphology ,adaptive method are used. By using the K-means faster detection, in previous studies[24]. Dataset used for the test data and training data using the DRIVE database.

Table 1. Comparison of Segmented Methods

\begin{tabular}{|c|c|c|}
\hline Authors & Methods & Accuracy $(\%)$ \\
\hline $\begin{array}{l}\text { S. Sekhar et al. } \\
{[10]}\end{array}$ & Hough Transform & 94.7 \\
\hline $\begin{array}{l}\text { Deepali A. } \\
\text { Godse et al. } \\
\text { [11] }\end{array}$ & Thresholding, calculus & 98.45 \\
\hline $\begin{array}{l}\text { Sinthanayothin } \\
\text { et al. }[12]\end{array}$ & Gaussian blob & $\begin{array}{c}\text { 99.1(for OD) } \\
\text { 83.3(for vessels) }\end{array}$ \\
\hline $\begin{array}{c}\text { Nagveena et al. } \\
{[14]}\end{array}$ & $\begin{array}{l}\text { Adaptive median } \\
\text { thresholding }\end{array}$ & 91 \\
\hline $\begin{array}{l}\text { Roychowdhury } \\
\quad \text { et al . [15] }\end{array}$ & $\begin{array}{l}\text { high pass filtering, } \\
\text { post-processing }\end{array}$ & 95.2 \\
\hline $\begin{array}{c}\text { Ishmeet Kaur1 } \\
\text { et al. }[16]\end{array}$ & $\begin{array}{c}\text { Fuzzy c-means, } \\
\text { neutrosophic approach }\end{array}$ & 98.74 \\
\hline $\begin{array}{c}\text { Franklin et al. } \\
\text { [17] }\end{array}$ & $\begin{array}{l}\text { multilayer perceptron } \\
\text { neural network }\end{array}$ & 95.03 \\
\hline
\end{tabular}




\begin{tabular}{|c|c|c|}
\hline $\begin{array}{c}\text { Roberto } \\
\text { Annunziata } \text { et } \\
\text { al. }[18],\end{array}$ & $\begin{array}{c}\text { Hessian-based, ad hoc } \\
\text { exudate inpainting }\end{array}$ & 95.62 \\
\hline Li et al. [19] & Model based approach & 99 \\
\hline $\begin{array}{c}\text { Huajun Ying } \text { et } \\
\text { al. } \text { [20] }\end{array}$ & $\begin{array}{c}\text { Local histogram } \\
\text { analysis }\end{array}$ & Not Reported \\
\hline $\begin{array}{c}\text { Lalonde } \text { et al. } \\
{[21]}\end{array}$ & $\begin{array}{c}\text { pyramidal } \\
\text { decomposition, } \\
\text { Handoff-distance }\end{array}$ & Not Reported \\
\hline $\begin{array}{c}\text { Roychowdhury } \\
\text { et al [22] }\end{array}$ & Gaussian Mixture \\
Model & 100 \\
\hline $\begin{array}{c}\text { Adithya } \text { et al. } \\
\text { [23] }\end{array}$ & $\begin{array}{c}\text { K-means clustering, } \\
\text { Adaptive morphology }\end{array}$ & Not Reported \\
\hline
\end{tabular}

http://messidor.crihan.fr/download-en.php

[7] Siddalingaswamy C , Gopalakrishna rabhu K , "Automatic localization and boundary detection of optic disc using implicit active contours", Internation Journal of Computer Applications, vol. 1, no. 7, pp. 1-5, 2010.

[8] Michael D. Abramoff, Meindert Niemeijer, "The automatic detection of the optic disc location in retinal images using optic disc location regression", Conf roc IEEE Eng Med Biol Soc , 1: pp 44 2- 4435, 2006.

[9] Jaspreet Kaur, Dr Sinha, "Automated localization of optic disc and macula from fundus images", International Journal of Advanced Research in Computer Science and Software Engineering, vol. 2, Issue 4, pp. 242-249, April 2012.

[10] S. Sekhar, W. Al-Nuaimy , A K Nandi, "Automated localization of retinal optic disc using Hough transform", pp 1577-1580, IEEE 2008

\section{CONCLUSION}

Automatic extraction of retinal blood vessels and localization of optic disk are very important and necessary for the detection of diseases in the eyes. It is helpful for early diagnosis of diseases from ophthalmic images. Retinal image suffers from various factors which may drop off the accuracy of extraction of blood vessels. In this paper, various methods of localization of optic disc are discussed. Besides some blood vessel segmentation techniques are also comprehensively discussed.

Very few methods have been worked on segmentation techniques that are independent of difference of blood vessels and optic disc, so more work can be done in this field too. This work can be useful for various retinal disease detection techniques and also useful for future research works.

\section{ACKNOWLEDGMENTS}

Authors wishes to acknowledge each and every individual who have supported for the current work directly or indirectly. We would like to thank our guide Ms Remya P S, Assistant Professor in the Department of Computer Science And Engineering, for valuable guidance. Our sincere thanks to Lord Almighty, who has been guiding us in every step.

\section{REFERENCES}

[1] Research Section, Digital Retinal Image for Vessel Extraction (DRIVE) Database. Utrecht, The Netherlands, Univ. Med. Center Utrecht, Image Sci. Inst. [Online]. Available:http://www.isi.uu.nl/Research/Dtabase/DR.

[2] STARE Project Website. Clemson, SC, Clemson

[3] T. Kauppi, V. Kalesnykiene, J.-K. Kamarainen, L. Lensu, I. Sorri, H. Uusitalo, H. Klviinen, and J. Pietil, "Diaretdb0: Evaluation database and methodology for diabetic retinopathy algorithms," Lappeenranta. University of Technology, Finland, Tech. Rep., 2006.

[4] T. Kauppi, V. Kalesnykiene, J.-K. Kmrinen, L. Lensu, I. Sorr, A. Raninen, R. Voutilainen, H. Uusitalo, H. Klviinen, and J. Pietil, "Diaretdb1 diabetic retinopathy database and evaluation protocol," Proc. of the 11th Conf. on Medical Image Understanding and Analysis (MIUA2007), pp.61-65, 2007.

[5] K. U. Research, "Chase db1," January 2011. [Online]. Available:http://blogs.kingston.ac.uk/retinal/chasedb1/.

[6] "Messidor: Digital retinal images." [Online]. Available:

[11] Deepali A. Godse. Dr. Dattatraya S. Bormane, "Automated Localization of Optic Disc in Retinal Images", International Journal of Advanced Computer Science and Applications, Vol. 4, No. 2, 2013.

[12] C. Sinthanayothin, J Boyce, Cook and T Williamson, "Automated localization of the optic disc, fovea and retinal blood vessels from digital color fundus images", Br J Ophthalmol, 83: pp. 902-910, Feb. 1999.

[13] Meindert Niemeijer, Michael D. Abramoff, Bram Van Ginneken, "Segmentation of the optic disc, macula and vascular arch in fundus photographs", IEEE Transactions on medical Imaging, vol.26, no.1, pp. 116-127, January 2007.

[14] Nagaveena, Deepashree Devaraj, Dr. S. C. Prasanna Kumar, " Vessels Segmentation In Diabetic Retinopathy By Adaptive Median Thresholding ", The International Journal Of Science \& Technoledge (ISSN 2321 - 919X).

[15] S. Roychowdhury, D. Koozekanani, and K Parhi,."Blood Vessel Segmentation of Fundus Images by Major Vessel Extraction and Sub-Image Classification", IEEE Journal of Biomedical and Health Informatics IEEE J..Biomed. Health Inform., pp. 1-1,2014.

[16] Ishmeet Kaur1, Lalit Mann Singh, " A Method of Disease Detection and Segmentation of Retinal Blood Vessels using Fuzzy C-Means and Neutrosophic Approach", Imperial Journal of Interdisciplinary Research (IJIR),Vol-2, Issue-6, 2016.

[17] S. W. Franklin and S. E. Rajan,."Computerized screening of diabetic retinopathy employing blood vessel segmentation in retinal images", Biocybernetics and Biomedical Engineering, vol. 34, no. 2, pp. 117124,2014 .

[18] Roberto Annunziata, Andrea Garzelli, Lucia Ballerini, Alessandro Mecocci,and Emanuele Trucco, "Leveraging Multiscale Hessian-Based Enhancement With a Novel Exudate Inpainting Technique for Retinal Vessel Segmentation", IEEE journal of biomedical and health informatics, vol. 20, no. 4, july 2016.

[19] $\mathrm{Li}$ and O Chutatape, "Automated feature extraction in color retinal images by a model based approach", IEEE Trans Biomed Eng, vol. 51, no.2, pp. 246-254, Feb 2004. 
[20] Huajun Ying, Ming Zhang and Jyh-Charn Liu, "Fractalbased automatic localization and segmentation of optic disc in retinal images", IEEE Proc. of the 29th Annual International conference of the IEEE EMBS, France.

[21] M Lalonde, M Beaulieu and Langis Gagnon, "Fast and robust optic disc detection using pyramidal decomposition and Hausdorff-Based Template Matching", vol. 20, No.11, pp. 1193-2001, Nov. 2001.

[22] Sohini Roychowdhury, Dara D. Koozekanani, Sam N. Kuchinka, and Keshab K.Parhi "Optic Disc Boundary and Vessel Origin Segmentation of Fundus Images", IEEE Journal of Biomedical and Health Informatics.2015.
[23] Adithya Kusuma Whardana, Nanik Suciati, " A Simple Method for Optic Disk Segmentation from Retinal Fundus Image ", I.J. Image, Graphics and Signal Processing, 2014, 11, 36-42.

[24] Youssif, Aliaa Abdel-Haleim Abdel-Razik, Atef Zaki Ghalwash, and Amr Ahmed Sabry Abdel-Rahman Ghoneim. "Optic Disc Detection From Normalized Digital Fundus Images by Means of a Vessels' Direction Matched Filter." IEEE TRANSACTIONS ON MEDICAL IMAGING 27.1 (2008): 11.

[25] www.google.co.in 